\title{
Uterine Cervical Smear Patterns In Females Attending A Teaching Hospital In Rural Eastern Uttar Pradesh, INDIA.
}

\author{
Dr. Aarti B.Bhattacharya ${ }^{1}$, Dr. Anjana Agarwal ${ }^{2}$, Dr. A D Dwivedi ${ }^{3}$ \\ Dr Shamima Khan ${ }^{4}$, Dr.Rumpa Das ${ }^{5}$, Dr.Sangita Bohara ${ }^{6}$, Dr.Ritu Sharma \\ ${ }^{1}$ Professor, Pathology, ${ }^{2}$ Professor \& ${ }^{3}$ Professor \& HOD-Obstetrics \& Gynecology. ${ }^{4,5,6,7}$ Assistant Professor, \\ Pathology, Hind Institute of Medical Sciences, Safedabad. Barabanki (Up) India.
}

\begin{abstract}
:
Introduction: A conventional Pap Test is the mainstay screening system for cervical cancer in a low resource setting in a developing country like India.In order to counsel women and to organize a cervical cancer screening programme by Pap test it is imperative to know the existing pattern of premalignant and malignant lesions here.

Materials and Methods: The present prospective and retrospective study was undertaken with the aim of evaluation of the abnormal cytology detected by cervical Pap smear study among patients reporting to out door Department of Obstetrics \& Gynaecology, Hind Institute Of Medical Sciences. Safedabad, Barabanki Uttar Pradesh, India. This study included cases from Jan 2015 to May 2016. It included all new cases attending Obstetric \& Gynaecology OPD. Total 1050 samples were included in the study period, of which 10 were unsatisfactory. The prevalence of epithelial cell abnormality in the pap smear was calculated in percentages. Specimen adequacy and the reporting were based on the revised Bethesda system 2001.

Results: Out of 1050 smears, 1040 smears were satisfactory.18 (1.7\%) revealed epithelial cell abnormality. ASCUS was detected in $02(0.19 \%)$ cases, low grade lesions were detected in 10 (0.96\%) cases, $05(0.48 \%)$ cases revealed high grade lesions and 01 (0.096\%) case was malignant. Low grade lesions (LSIL/CINI) belonged to 30-35 years age group. High grade lesions (H SIL / CIN II) were found in 40-45 age group.Severe Dysplasia (HSIL/CINIII)was found in the age group of 45-50 years. Invasive carcinoma, diagnosed in a case aged 70 years. The bulk of high grade lesions being diagnosed in patients of age 45 or above. $25 \%$ of the patients (131.25) with an abnormal Pap smear showed healthy cervix on per vaginal examination.This very fact endorses the utmost importance of a regular Pap Test.

Conclusion: Cervical carcinoma is prevalent more amongst the economically backward strata because of lack of awareness about the disease, about the cervical cancer screening programme, and lack of awareness amongst the health workers. Cancer cervix has a prolonged premalignant phase and this fact makes invasive carcinoma cervix a preventable condition.
\end{abstract}

Keywords: Cervical smear, Cervical carcinoma, LSIL, HSIL, Pap test

\section{Introduction}

Mention of cervical smear cytology and biopsy were made in literature as early as the $19^{\text {th }}$ century [1] .It was realized that cervix biopsy helps in detecting premalignant and malignant lesions of cervix. [2] .Rubin in 1910 mentioned about the structural changes in the cervical epithelium surrounding invasive cancer. These structural changes were considered as a manifestation of early cervical cancer [3]. It was George Papanicoloau who ushered in the golden era of Pap smear cytology in the year 1920. Cervical cancer is considered preventable as the premalignant stages can be detected easily by studying the exfoliated cells (Papanicoloau smear) [Pap Test]. A conventional Pap smear is being used in low resource settings, in developing countries for cervical cancer screening. In higher centres and Western countries liquid based cytology with Human Papilloma Virus (HPV) PCR testing is used for cervical cancer screening.[5]

Cervical epithelial cell abnormalities in the Pap smear represent a spectrum of intraepithelial lesions from mild to severe dysplasia to invasive carcinoma. According to the 2001 revised Bethesda System for reporting cervical cytology, epithelial cell abnormalities originate in the squamous or glandular cells of the cervix.

The category of atypical squamous cells (ASC) includes ASC-US (ASC of undetermined significance) and ASC-H (ASC, cannot exclude high grade squamous intraepithelial lesions). The positive predictive value for H SIL (High Grade Squamous Intraepithelial lesion) in ASC-H is higher than in ASC-US, but not as high as in the category of H SIL. The generic term squamous intraepithelial lesion 
( SIL) is subtyped into L SIL-low grade squamous intraepithelial lesion (lesions showing perinuclear halo along with mild dysplasia) and, H SIL =High grade squamous intraepithelial lesion -( lesions showing moderate to severe dysplasia and carcinoma in situ). The category of atypical glandular cells is designated as AGC. Smears showing no epithelial abnormalities are specified as Negative for intraepithelial lesion or malignancy (NILM) in the revised Bethesda System.[3]

Pap smear screening has lead to a drastic reduction in the incidence of invasive cervical cancer in many countries across the world. Various studies show that a majority of cervical cancer mortality is from the low and middle income group of countries. This may be attributed to the absence of an efficient cervical cancer screening system in the developing countries.[8,13]

Cervical smears have become a valuable tool in screening for cervical lesions even at rural peripheral centres because of its easy availability, cost effectiveness, sensitivity, specificity and reliability.

This present study was undertaken to evaluate the abnormal cytological entities detected in Pathology Department by cervical Pap smears examination among patients reporting to the outdoor Dept of Obstetrics \& Gynaecology in a Teaching Hospital in rural Eastern UP,INDIA.The cervical smear patterns would be correlated with clinical profile of patients. The findings would be correlated with duration of sexual activity, parity, menopause and significance would be derived.

\section{Materials and Methods}

This study was carried out prospectively and retrospectively over a period of one year, five months (Jan.2015 to May.2016).The exclusion criteria used were - already diagnosed cases, cases undergoing treatment for cancer cervix and postoperative cases.

Detailed history was obtained with special mention of age, parity and complaints. Perspeculum findings were noted. Cervical smear received in Ether-Alcohol fixative was stained by Papanicolaou stain and examined by two different experienced Pathologists using the revised Bethesda system. In some instances wherever indicated and feasible, subsequent cervical biopsy was taken and studied under light microscope. Thus the cyto-histopathological findings were compiled and correlated in as many cases as possible.

\section{Results}

In this study 1050 smears of 525 patients were studied Out of this total, 1040 were satisfactory and were evaluated. 400 patients $(76.19 \%)$ were from reproductive age group in between $21-50 \mathrm{yrs}$. Cases below $20 \mathrm{yrs}$ were $10(1.90 \%)$ and cases above $50 \mathrm{yrs}$ were $115(21.90 \%)$. Maximum cases (330) were in 25-40 yrs age group (62.85\%).

Table no: 1 Showing Present Study Details.

\begin{tabular}{|l|c|}
\hline Study Period & Jan 2015 to May 2016 \\
\hline Total number of patients & 525 \\
\hline Total No: of Pap smears & 1050 \\
\hline No: of satisfactory smears & 1040 \\
\hline No: of unsatisfactory smears & 10 \\
\hline
\end{tabular}

The patients with unsatisfactory smears were advised to visit again for repeat Pap Test but unfortunately were lost to follow-up.

Table no: 2 Showing patient characteristics in the present study.

\begin{tabular}{|l|c|c|}
\hline Patient's Age group & No: of women & Percentage \\
\hline $21-50$ yrs(Reproductive grp) & 400 & $76.19 \%$ \\
\hline Below 20 yrs & 10 & $1.90 \%$ \\
\hline Above 50 yrs & 115 & $21.90 \%$ \\
\hline Total No: of women & 525 & $100 \%$ \\
\hline
\end{tabular}


Table No: 3 Showing clinical complaints of women under study.(n-525)

\begin{tabular}{|l|c|c|}
\hline \multicolumn{1}{|c|}{ Symptoms } & Number of women & Percentage \\
\hline 1.Vaginal discharge & 105 & $20 \%$ \\
\hline 2.Pain in abdomen & 60 & $11.42 \%$ \\
\hline 3.Menstrual irregularity & 60 & $11.42 \%$ \\
\hline 4.Bleeding per vaginum & 120 & $22.85 \%$ \\
\hline 5.Mass coming out of vagina & 35 & $6.67 \%$ \\
\hline 6.Infertility & 35 & $6.67 \%$ \\
\hline 7.Dyspareunia & 80 & $15.23 \%$ \\
\hline \multicolumn{1}{|c|}{ Total } & 495 & $94.29 \%$ \\
\hline
\end{tabular}

Table No: 4 Showing study of asymptomatic females. $(n=30)$

\begin{tabular}{|l|c|c|}
\hline Examination Findings & No: of females & Percentage \\
\hline 1Cervical polyp & $\mathbf{0 2}$ & $\mathbf{6 . 6 7 \%}$ \\
\hline 2 Erosion & $\mathbf{0 4}$ & $\mathbf{1 3 . 3 3 \%}$ \\
\hline 3.Non significant findings & $\mathbf{2 4}$ & $\mathbf{8 0} \%$ \\
\hline Total No: of women & 30 & $\mathbf{1 0 0} \%$ \\
\hline
\end{tabular}

Table No: 5 Showing classification of NILM cases according to The revised Bethesda System. $(\mathbf{n}=1040)$

\begin{tabular}{|c|c|c|}
\hline Microscopic findings & No: of cases & $\begin{array}{c}\text { Percentage } \\
\text { \% }\end{array}$ \\
\hline Reactive cellular changes & $\mathbf{0 8}$ & $\mathbf{0 . 7 6 9 \%}$ \\
\hline Infections: & & $\mathbf{0 . 0 9 6 \%}$ \\
\hline Trichomonas Vaginalis & $\mathbf{0 1}$ & $\mathbf{7 . 6 9 \%}$ \\
\hline Bacterial vaginosis & $\mathbf{8 0}$ & $\mathbf{0 . 0 9 6 \%}$ \\
\hline Candida infection & $\mathbf{0 1}$ & $\mathbf{1 . 9 2 \%}$ \\
\hline Atrophic smears & $\mathbf{2 0}$ & $\mathbf{8 7 . 6 9 \%}$ \\
\hline No significant findings & $\mathbf{9 1 2}$ & \\
\hline & & \\
\hline
\end{tabular}

Table No: 6 Showing analysis of smears with epithelial cell abnormalities. $(n=1040)$

\begin{tabular}{|c|c|c|}
\hline Microscopic findings & Number of cases & Percentage \% \\
\hline ASCUS & $\mathbf{0 2}$ & $\mathbf{0 . 1 9} \%$ \\
\hline L SIL & $\mathbf{1 0}$ & $\mathbf{0 . 9 6} \%$ \\
\hline H SIL & $\mathbf{0 5}$ & $\mathbf{0 . 4 8} \%$ \\
\hline SCC invasive & 01 & $\mathbf{0 . 0 9 6 \%}$ \\
\hline
\end{tabular}




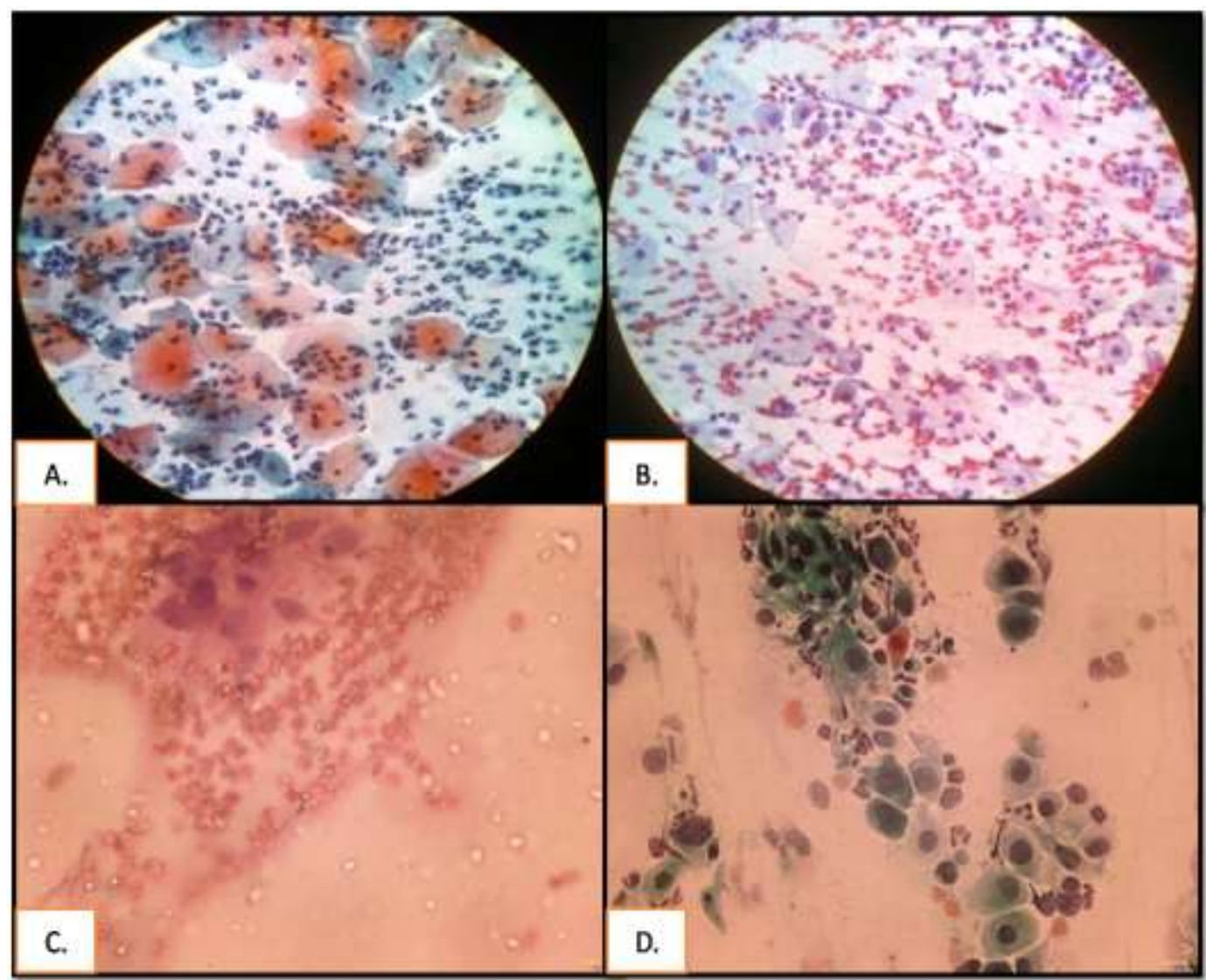

A.Reactive cellular changes Pap 40X B.LSIL.Pap 10X

C.HSIL Pap40X

D.SCC Pap 40X.

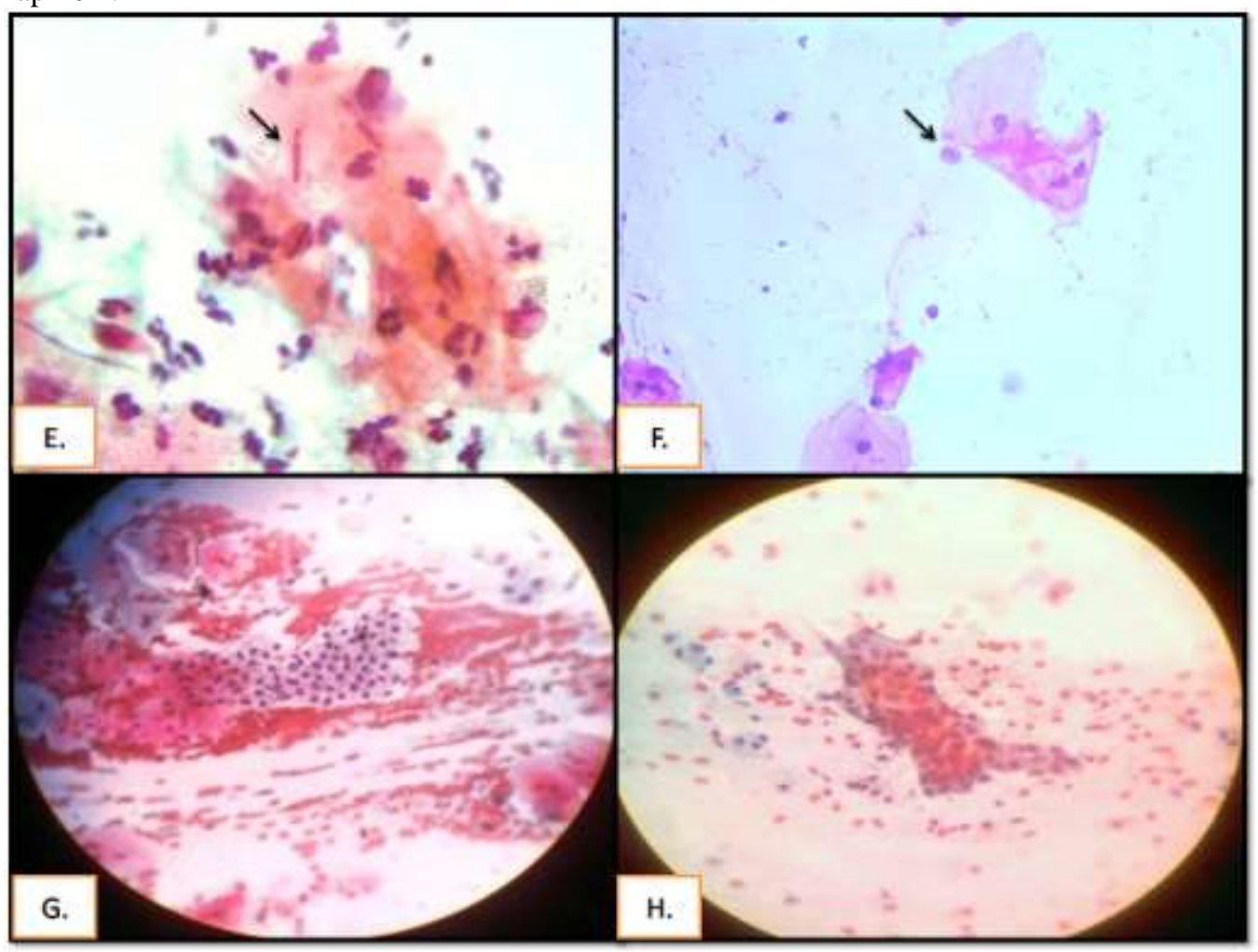

E.Candida Pap 40X

F.Trichomonas Vaginalis Pap 40X
G.Cervical erosion Pap 10X

H.Squamous metaplasia Pap 10X 


\section{Discussion}

Cervical cancer is still a major health problem in the economically backward countries of the world. There are studies regarding the Pap smear patterns from various areas. Our study was one such attempt to study the existing pattern here as of now. Edelmen et al [12] studied 29,295 females in one year and found ASCUS in $9.9 \%$, L SIL in $2.5 \%$, H SIL in $0.6 \%$, invasive carcinoma cervix in $0.2 \%$. In a study conducted by Urmila Banik et al [3] in Bangla Desh in 2010 they found ,ASCUS $-0.18 \%$, AGC$0.12 \%$, L SIL- $6.36 \%, \mathrm{H}$ SIL-1.18 \% , and malignancy $-0.35 \%$. According to another study in Brazil [10] on $18,22,441$ pap smears in 5 years, L SIL was found in the age group of 15-30 years, high grade lesions including cancer cervix were found in women aged 40 years and above. According to our study ASCUS was found in $0.19 \%$, L SIL was found in $0.96 \%$ in the age group of 35-39. H SIL,Cervical carcinoma in situ were found in $0.48 \%$ in the age group of 40-45 and above. Case of invasive cancer cervix was found in $0.096 \%$ women aged 70 years. Previous studies indicate that premalignant lesions occur around 35-40 years. Both low grade and high grade lesions are common in women aged 45 years and above because they are the under users of the cervical cancer screening programme. Our study also reiterates the same findings. Thus it was found that as age of the patients increased the incidence of lowgrade and high-grade squamous lesions and carcinoma cervix also increased with the relationship being statistically significant.The occurance of these lesions also showed an association with increasing parity and period of sexual activity.

\section{Conclusion}

Cervical carcinoma is prevalent more amongst the economically backward strata. Cervical cancer a leading cause of morbidity and mortality can be prevented through simple costeffective measures like creating awareness and regular screening with Pap smear in the premenopausal and postmenopausal age groups. Conventional cytology or the Pap smear is a cost effective and efficient method for screening of patients .Cancer cervix has a prolonged premalignant phase and this fact makes invasive carcinoma cervix a preventable condition. The average life expectancy of people has increased so there is a possibility that a lingering low grade lesion might transform into a high grade lesion and invasive carcinoma. This makes it necessary for every hospital to make the cervical screening programme available to every woman more so in the vulnerable age group. A raised prevalence of epithelial cell abnormality reflects the lack of awareness among people about cervical cancer screening programme. $[8,13]$ Seeing to this it becomes the duty of every governmental and non-governmental agency to sensitize the mass regarding this preventable cancer of cervix.

\section{References}

[1] Coleman DV, Evans D. Introduction : Biopsy pathology and cytology of cervix.Ist edition, Chapman and Hall, London 1988:1-6.

[2] Rubin IC . The pathological diagnosis of incipient carcinoma of the uterus .Am J Obst, 1910;62:668 - 676.

[3] Urmila Banik,Pradip Bhattacharjee,Shahabuddin Ahmad,Zillur Rehman:Pattern of epithelial cell abnormality in Pap smear:a clinicopathological and demographic correlation. Cyto Jr 2011,8;8.

[4] Misra JS ,Srivastava S,Singh U,Srivastava A N. Risk factors and strategies for control of carcinoma cervix in India:Hospital based cytological screening experience of 35 years. Indian Journal of Cancer,2009,46(2);155-159.

[5] Bhatla N, Mukhopadhyay, Kripalani A Pandey R M Pandey R, Gravitt P E Shah K V ,Iyer VK,Verma K.Evaluation of adjunctive tests for cervical cancer screening in low resource settings. Indian Journal of Cancer, 2007: 44 (2): 51 - 55.

[6] Maryam Ansari, Ghazala Mehdi, SH Arif ,Hena Ansari ,Tankin Khan. Smear patterns and spectrum of premalignant and malignant cervical epithelial lesions in postmenopausal Indian women: A hospital based study: Diagnostic Cytopathology 2012Nov,4D(11):976-83.

[7] Toews HA .The abnormal pap smear. A rationale for follow up. Can F am Physician 1983, 29759 - 62

[8] Suba FJ, Raab S S Viet / American Cervical Cancer Prevention Project. Papanicolaou screening in developing countries. An idea whose time has come. Am J Clin Pathol .2004; 121:315 - 20.

[9] Cho H, Kim J H. Treatment of the patients with abnormal cervical cytology. 'A see and treat versus three -step strategy. J Gynecol Oncol 2009; $20: 164-8$.

[10] Kapila K, George S S, A 1- Shaheena, A 1- Ottibi M S ,Pathan S K, Sheikh Z A ,et al .Changing Spectrum of Squamous Cell Abnormalities Observed on papanicolaou smears in Mubarak Al - Kabeer Hospital, Kuwait, over a 13 - year Period .Med. Pnic pract 2006; 15: 253 - 9.

[11] Abdullah L S .Pattern of abnormal Pap smears in developing countries. A report from a large referral hospital in Saudi Arabia using the revised 2001 Bethesda System. Ann. Saudi Med $2007 ; 27: 268-72$.

[12] Edelman M, Fox A .Cervical Papanicolaou smear abnormalities in inner Bronx adolescents: prevalence, progression and immune modifiers.Cancer (cancer cytopathology) 1999;87: 184 - 9.

[13] Bishop A,Wells E, Sherris J, Tsu V ,Cook B .Cervical cancer; Evolving prevention strategies for developing countries. Reprod. Health Matters. 1995; $6: 60-7$. 